\title{
Clostridium difficile toxin assay by purified specific antitoxins coated to latex beads
}

\author{
Chetana Vaishnavi, Akshita Kapila, Meenakshi Singh, Kartar Singh \\ Department of Gastroenterology, \\ Postgraduate Institute of Medical Education and Research, Chandigarh, 160012, India.
}

\begin{abstract}
Backround \& Objective: Clostridium difficile associated disease (CDAD) is a matter of grave concern in the hospital environments due to antimicrobial usage.

Methods: We investigated the clinical and demographic profile of patients whose fecal samples were received in our laboratory and correlated the same with their $C$. difficile toxin (CDT) status. Six hundred twenty nine consecutive and non-repeat fecal samples were subjected to CDT assay using purified anti-toxin A and anti-toxin B coated to latex beads. Semi-quantitative titrations were carried out with the positive samples with a doubling dilution method. Clinical and demographic profile of each patient was recorded. During analysis the patients were assigned to two groups (i) Group 1 comprised of those receiving antibiotics and/or other drugs and (ii) Group 2 of those not receiving any drug.
\end{abstract}

Results: The age of the patients ranged from a few days to 93 years. Predominant clinical symptoms were diarrhoea (98.7\%), abdominal pain (35.9\%) and fever (49.8\%). CDT was positive in $45.8 \%$ with titers ranging from 1 in 5 to 1 in 2560. CDT positivity was highly influenced by prior antibiotic and drug intake $(\mathrm{p}<0.05)$. Fever was present in $43.4 \%$ and abdominal pain in $35.5 \%$ of CDT positive cases. CDT positivity was also significantly associated with age below 2 years $(\mathrm{p}<0.001)$ and between $41-55$ years $(\mathrm{p}<0.01)$. CDT positivity was highly associated with gastrointestinal diseases $(32.5 \%)$ and age.

Interpretation \& conclusion: Readily available clinical and basic laboratory data are useful for correlation with severity of CDAD.

Keywords: Clinical presentation, Clostridium difficile, patient demographics, toxin assay.

\section{INTRODUCTION}

Clostridium difficile is recognized globally as an important enteric pathogen associated with considerable morbidity and mortality. Widespread outbreaks of CDAD are increasingly being reported worldwide and is believed to be due to the broad-spectrum antimicrobial use ${ }^{1,2}$ as well as the use of other drugs such as proton pump inhibitors, ${ }^{3}$ immunosuppressives ${ }^{4}$ and cancer therapeutics. ${ }^{5,6}$ The elderly and the debilitated patients

\section{Corresponding Author :}

Dr. C. Vaishnavi, Professor (GE Microbiology)

Department of Gastroenterology

PGIMER, Chandigarh, India.

Tel: 91-172-2756609

Fax: 91-172-2744401; 2745078

Email:cvaishnavi@ rediffmail.com chetanavaishnavi@gmail.com easily contract the disease predominantly due to the overzealous use of antibiotics. ${ }^{2,7}$

The role of the hospital was not clearly demarcated in earlier epidemiologic studies of C.difficile. ${ }^{8} \mathrm{As}$ awareness increased; various symptoms present during CDAD were taken into consideration to diagnose the disease clinically. Thus profuse watery, green foul smelling or bloody diarrhoea along with abdominal cramps and fever is regarded as the hallmark of CDAD. Even though diarrhoea is generally a side effect of many commonly used antibiotics, the overgrowth of drug resistant $C$. difficile - either endogenous in origin or acquired exogenously - can result in nosocomial diarrhoea.

C. difficile produces two highly lethal exotoxins toxin A and toxin B. Toxin A is primarily an enterotoxin and is associated with extensive damage to the gastrointestinal wall and accumulation of luminal fluid ${ }^{9}$ even though it is also a cytotoxin. Toxin B is by and large 
a cytotoxin that appears to come into play after the damage is done by toxin A. ${ }^{10}$ Both the toxins open up the tight junctions between the intestinal epithelial cells of the gut and hence aid vascular permeability and also cause haemorrhage. ${ }^{11}$

Production of toxins A and B by $C$. difficile is an important factor for CDAD manifestation and the diagnosis of CDAD is based primarily on the detection of these toxins. The combination of the presence of C. difficile in hospitals and the number of people receiving antibiotics and other drugs in these settings can lead to frequent outbreaks of CDAD.

We investigated the clinical and demographic profile of patients whose fecal samples were received in our laboratory and correlated the same with their status. The study was approved by the Ethical Committee of the Institute.

\section{MATERIALS AND METHODS}

(i) Patient population and sampling: Six hundred and twenty nine consecutive and non-repeat fecal samples from indoor and out door patients were received in the Microbiology Division, Department of Superspecialty of Gastroenterology, with specific request for $C$. difficile toxin (CDT) assay during the study period ranging from April 2007 to March 2008. The patients whose samples were received belonged to various medical, surgical and emergency departments of Postgraduate Institute of Medical Education and Research, Chandigarh, India. This tertiary care centre is a referral hospital which caters to patients coming from entire north-west India and beyond.

(ii) C. difficile toxin assay: $C$. difficile toxins A and B were detected in the fecal specimens as described earlier ${ }^{3,12}$ using purified anti-toxin A and anti-toxin B (kindly provided by Dr. M. Warny, USA). Briefly, 50 $\mu 1$ of 1 in 5 diluted fecal supernatant was taken on a clean glass slide to which ready-to-use $C$. difficile antitoxin A or B coated latex beads were added. The slide was gently rocked manually and checked for macroscopic agglutination. The fecal supernatant that agglutinated instantly with latex beads coated with anti-toxin A was taken to be positive for toxin A and that which agglutinated with anti-toxin $B$ as positive for toxin B. A known positive fecal sample obtained from a patient with antibiotic associated diarrhea served as the positive control. Two negative controls consisted of (i) an unreactive fecal sample from a healthy subject who had no antibiotic exposure for 6 weeks prior to testing and (ii) uncoated latex beads plus diluted test sample. All positive samples were further subjected to titrations by doubling dilutions of the samples and repeating the procedure. The toxin titer was determined by a positive agglutination reaction with the highest dilution of the fecal supernatant.

(iii) Demographic analysis: The laboratory records were reviewed for patient demographics, clinical presentation, medical history, therapy etc. Patients of all age groups were included in the study. All prescriptions for antibiotics and other drugs were taken into consideration. During the time of analysis the patients were assigned to two groups (i) Group 1 comprised of those receiving antibiotic and other drugs (RAD) and (ii) Group 2 of those not receiving any antibiotic or drug (NRAD).

(iv) Statistical analysis: The data were expressed by descriptive statistics. The statistical analysis for comparison of RAD and NRAD groups and CDT positive cases in them was carried out by the Chisquare test for qualitative data. $\mathrm{p}<0.05$ was considered statistically significant difference.

\section{RESULTS}

(i) Clinical and demographic profile: Of the 629 cases analyzed, 398 (63.3\%) were males and 231 $(36.7 \%)$ females. The age of the patients ranged from a few days to 93 years. Predominant clinical symptoms present in them were diarrhoea (98.7\%), abdominal pain $(35.9 \%)$ and fever $(49.8 \%)$. The patients were undergoing treatment for various ailments such as hepatic, gastrointestinal, cardiac, pulmonary, renal and blood diseases, cancers, transplant and other surgeries. There were 486 (77.3\%) patients in RAD group and $143(22.7 \%)$ in NRAD group. Most common antibiotics received by the patients in RAD group were metronidazole, vancomycin, cephalosporins, ciprofloxacin and meropenem. Apart from antibiotics, other drugs received by them included proton pump inhibitors (PPI), steroids and chemotherapeutics.

(ii) CDT positive cases: $C$. difficile toxin was positive in $288(45.8 \%)$ of the total 629 samples of which both toxin $\mathrm{A}$ and $\mathrm{B}\left(\mathrm{A}^{+} \mathrm{B}^{+}\right)$were present in $89.6 \%$ whereas only toxin $\mathrm{A}\left(\mathrm{A}^{+} \mathrm{B}^{-}\right)$or $\mathrm{B}\left(\mathrm{A}^{-} \mathrm{B}^{+}\right)$was present 


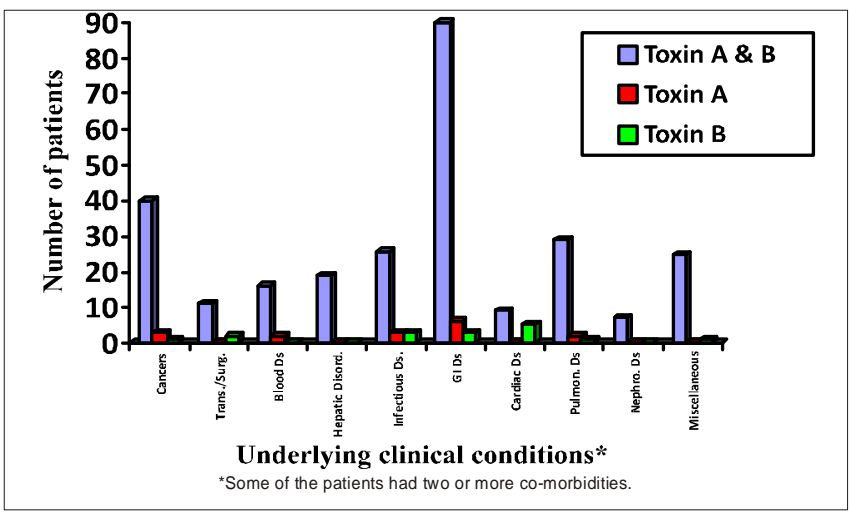

Fig. 1 : Fecal CDT positivity with respect to clinical conditions.

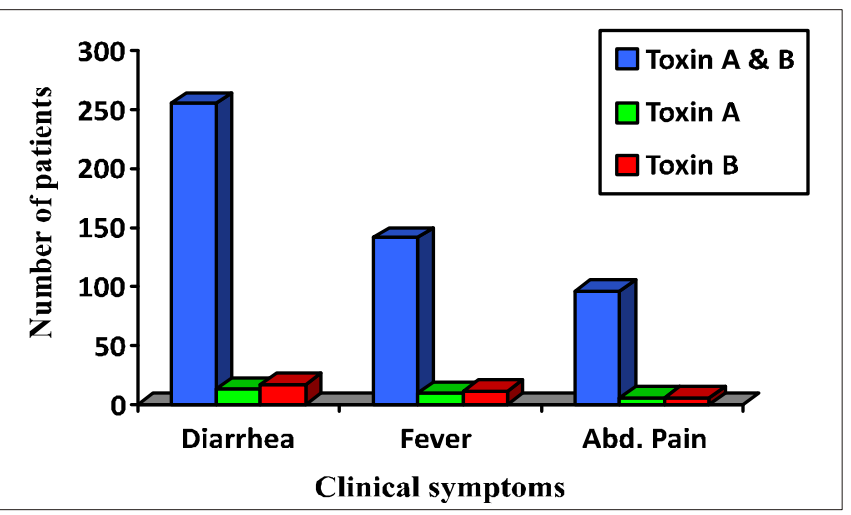

Fig. 2 : Fecal CDT positivity in relation to clinical symptoms.

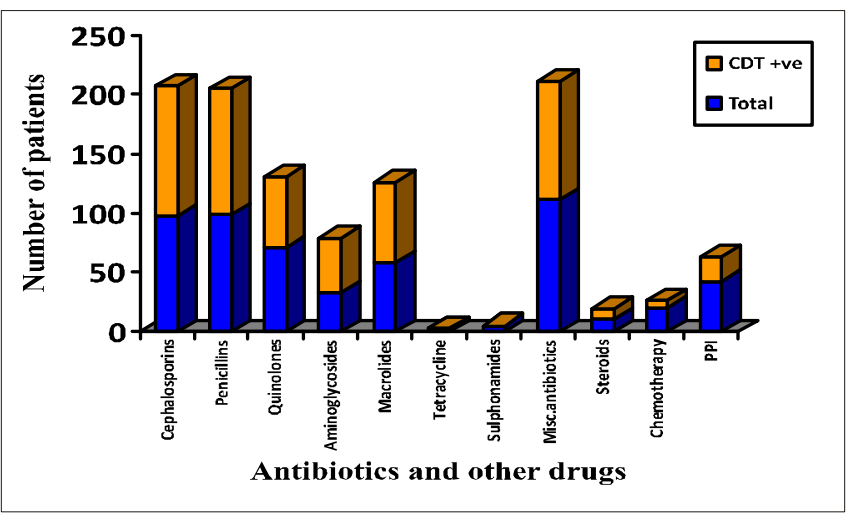

Fig. 3: Fecal CDT positivity in relation toantimicrobials and other drugs received.

in $4.5 \%$ and $5.9 \%$ respectively. The range of CDT titers for toxin A and B among the fecal samples $(\mathrm{n}=288)$ was from 1 in 5 to 1 in 2560 . The distribution of CDT positivity in regards to clinical conditions, clinical symptoms, intake of antibiotics and other drugs respectively are shown in Figs 1-3. CDT positivity was influenced by prior antibiotic and other drug usage as $81.3 \%$ patients in RAD group were CDT positive as compared to only $18.7 \%$ in NRAD group $(\mathrm{p}<0.05)$. The antibiotics that were more commonly associated with CDT positivity in decreasing order were cephalosporins, penicillins, quinolones, macrolides and aminoglycosides. Amongst the patients with other drug exposure, PPI use was more commonly associated with CDT positivity.

(iii)Relationship of CDT positivity with various factors: CDT positivity was higher in males $(47.6 \%)$ compared to females $(42.6 \%)$ but it was not significantly associated $(p>0.05)$ with gender. Age group analysis for 2-sided test showed significant association with CDT positivity with age group below 2 years $(\mathrm{p}<0.001)$ and between 41-55 years $(\mathrm{p}<0.01)$. CDT positivity was highly associated with gastrointestinal diseases $(32.5 \%)$ and in decreasing order with cancers, pulmonary diseases, infectious diseases, hepatic disorders and others. Diarrhoea was the most significant $(p<0.001)$ clinical symptom present in both the RAD and NRAD groups followed by abdominal pain in RAD group ( $\mathrm{p}<0.001)$ only. Diarrhoea was significantly associated with CDT positivity in both the groups $(\mathrm{p}<0.001)$. Watery stools were seen in 47.2\% CDT positive cases while $45.9 \%$ passed semisolid stool and 7\% passed solid stool. Apart from this, $7.6 \%$ of the CDT positive cases passed green colored stool and $14.9 \%$ passed blood in stool. However, none of these parameters were significantly associated with CDT positivity ( $p>0.05$ ). CDT was also positive in $43.4 \%$ cases with fever and $35.4 \%$ with abdominal pain. But abdominal pain was only significantly associated with CDT positivity in RAD group $(\mathrm{p}<0.001)$.

\section{DISCUSSION}

The reason why CDAD is the most common in hospital settings is mainly due to clustering of cases in hospitals and even within hospital wards. ${ }^{13}$ Diarrhoea leads to dissemination of the pathogen in the hospital environment where they exist for a long time due to spore formation. ${ }^{14}$ Elderly patients undergoing antibiotic therapy for surgical procedures are at great risk of acquiring the infection. Even young immunocompromised patients are at constant risk. Acquisition of $C$. difficile may either result in asymptomatic carriage or end in mortality for patients undergoing antibiotic therapy for unrelated diseases. ${ }^{15}$ The identification of patients at risk in a hospital will help the clinicians to promptly diagnose and manage CDAD. 
In the present investigation the clinical and demographic profile of 629 patients were reviewed and correlated with their CDT status in order to identify factors most strongly associated with CDAD in our setting. Even though CDT positivity was detected in all age group similar to our earlier report ${ }^{12}$ we found that age group below 2 years and between $41-55$ years were significantly associated with CDT positivity. The increased rates of CDAD amongst the middle age group in our setting could be due to various reasons inclusive of dissemination of the pathogen by silent reservoirs in the tertiary care hospital and multiple CDAD precipitating factors like underlying diseases and various drugs taken for the treatment. Though CDT positivity was higher in the males, it was not significantly associated with the gender, and might only reflect the increased opportunity for treatment amongst the males.

Antibiotic and other drug receiving group (RAD) was 3.47 times more prone to diarrhoea than the nonreceiving group (NRAD). Similarly the former group was found to be 1.16 times more prone to fever than NRAD. However, interestingly, abdominal pain was not found to be significant in RAD group compared to the NRAD group. Abdominal pain should therefore be regarded as a general clinical symptom of other underlying diseases and may or may not be present in CDAD during the time of testing in high risk patients. A high number $(45.8 \%)$ of the 629 samples were CDT positive and it was influenced by prior drug usage inclusive of antibiotics and proton pump inhibitors as $81.3 \%$ patients in RAD group were CDT positive compared to only $18.7 \%$ in the NRAD group ( $p<0.05$ ), although diarrhea was present in almost all of the CDT positive patients $(99.3 \%)$.

Amongst the factors that contribute to $C$. difficile pathogenicity, CDT production is the best characterized and the most important. ${ }^{16,17}$ Colonization of $C$. difficile may result in a broad spectrum of clinical conditions varying from non-specific diarrhoea to pseudomembranous colitis and CDT may be present in many of them. However the presence of CDT by itself may not necessarily be due to a case of CDAD, but patients having an antibiotic associated diarrhoea and who test positive for CDT are generally regarded to have CDAD., ${ }^{7,18}$ Earlier investigators worked primarily with toxin $A$ whereas others worked mostly with toxin B before the importance of both the toxins in the pathogenicity of CDAD was discovered. These workers may have missed some of the cases where $\mathrm{A}^{-} \mathrm{B}^{+}$or $\mathrm{A}^{+} \mathrm{B}^{-}$strains of $C$. difficile were the etiological factors. In the present study both toxins A and $B$ were detected in a large number of patients. Apart from these, toxin $\mathrm{A}^{+} \mathrm{B}^{-}$and $\mathrm{A}^{-} \mathrm{B}^{+}$cases were also detected in $4-6 \%$ of our samples.

Toxigenic isolates of $C$. difficile differ in amount of toxin production activity depending upon their serogroups. The risk of acquiring diarrhoea depends upon the infecting C. difficile strain. ${ }^{19}$ In the present study, data on the strains of $C$. difficile is not available. Other than this, CDAD rates and severity of the disease are also dependent on the host immune response and the levels of toxin neutralizing antibodies. In the present study, CDT titers ranged from 1 in 5 to 1 in 2560 in the CDT positive patients, with peak titers ranging from 1 in 20 to 1 in 80 . Akerlund et $a l^{20}$ investigated the relationship between fecal CDT levels in a CDAD endemic region and suggested that the broad range of fecal toxin levels observed may be due to coarse measure of diarrhea frequency and to the fact that toxin levels represented only one time point per patient during infection.

In the United States alone, it was estimated that there were about 3 million cases of CDAD every year with approximately 1 billion dollars being spent for healthcare expenses. ${ }^{2,21}$ Due to the prevalence of the global epidemic and hypervirulent strain, efforts should be made to isolate patients with CDAD to minimize the cross contaminations between patients. Identifying patients who are at high risk for severe CDAD early in the course of their infection may have clinicians responsibility and improve outcomes, but predictions are not well known ${ }^{22}$ and host factors are also likely to be predictors of illness and death. Das et $a l^{23}$ reported significantly higher mortality of patients with CDAD on glucocorticoids, compared to those without them, regardless of the severity of CDAD. Bajaj et $a l^{24}$ also reported that cirrhotics with CDAD have a higher mortality, longer length of hospital stay etc., compared with those without CDAD. Antibiotic and PPI use are risk factors for CDAD development in hospitalized cirrhotics. In our study, among the other drugs taken, PPI use was common and was seen to be associated with CDT positivity. Even among the underlying comorbidities, gastrointestinal diseases were the commonest, followed by malignancy, pulmonary diseases, infectious diseases and hepatic disorders. Halvorson et $a l^{25}$ reported an unusual case of fatal non-antibiotic associated CDAD following Salmonella serotype Saintpaul gastroenteritis in a previously healthy young person, re-emphasizing the risk of CDAD in the community. Thus studying toxin 
levels in feces influenced by both bacterial and patient factors, contribute to the severity of symptoms in CDAD patients.

Dubberke $e t a l^{26}$ suggest that only diarrhoea stools should be tested for $C$. difficile or its toxins. In case of non-diarrheal stools, review of patient records is required to ensure that patient has symptoms of $C$. difficile infection. ${ }^{27}$ Repeated stool testing for $C$. difficile positive cases serves no purpose unless the symptoms resolve with treatment. The degree and extent of $C$. difficile related paediatric diarrhoea might turn into a lot more worse condition than in adults. ${ }^{28}$ Infants are known to have a high number of toxigenic $C$. difficile and a high amount of toxin A and B in their stools and yet be completely asymptomatic. Such asymptomatic carriers produce a reservoir pool of the pathogen in the hospital environment. In the present study also a significant number of CDT positivity was seen among infants below 2 years.

Genetic subtyping, and binary toxin assays are currently not widely accessible, thereby making patient management difficult. Our results suggest that readily available clinical data such as age and basic laboratory data are correlated with severe CDAD. However, clinical symptoms like diarrhoea, fever and abdominal pain were not found to be significantly associated with toxin positivity, even though both toxin A and B were present in patients presenting with these clinical symptoms. Antibiotics like cephalosporins, penicillins, quinolones etc and drugs like PPI, immunosuppressives and chemotherapeutics were found to be associated with CDT positivity. Therefore hospital should support antibiotic policies that minimize the use of broad spectrum penicillins, cephalosporin and fluoroquinolones, as well as over the counter drugs like proton pump inhibitors.

\section{ACKNOWLEDGEMENT}

The work was carried out with the internal funds of the Institute. The authors wish to thank Dr. M. Warny, USA for donating purified anti-toxin A and anti-toxin B. The technical assistance of Ms. Monica Sharma and Mr. Babbar Raja and statistical evaluation by Ms. Kusum are also acknowledged.

\section{REFERENCES}

1. Hopkins MJ, MacFarlane GT. Changes in predominant bacterial populations in human faeces with age and with Clostridium difficile infection. J Med Microbiol 2002;51(5):448-54.

2. Blondeau, JM. What have we learned about antimicrobial use and the risks for Clostridium difficile-associated diarrhea? J Antimicrob Chemother 2009;63(2):238-42.

3. Kaur S, Vaishnavi C, Prasad KK, Ray P, Kochhar R. Comparative role of antibiotic and proton pump inhibitor in experimental Clostridium difficile infection in mice. Microbiol Immunol 2007;51(12):1209-14.

4. Kaur S, Vaishnavi C, Ray P, Kochhar R, Prasad KK. Effect of biotherapeutics on cyclosporin-induced Clostridium difficile infection in mice. J Gastroenterol Hepatol 2010;25(4):832-8.

5. Emoto M, Kawarabayashi T, Hachisuga T, Eguchi F, Shirakawa K. Clostridium difficile colitis associated with cisplatin-based chemotherapy in ovarian cancer patients. Gynecol Oncol 1996;61(3):369-72.

6. Kumar B, Vaishnavi C, Sandhu K, Kaur I. Clostridium difficile toxin assay in psoriatic patients. Trop Gastroenterol 2004;25(4):164-7.

7. Cherifi S, Delmee M, Broeck JV, Beyer I, Byl B, Mascart G. Management of an outbreak of $C$. difficile-associated disease among geriatric patients. Infect Control Hosp Epidemiol 2006;27(11):1200-5.

8. Bartlett JG. Historical perspectives on studies of Clostridium difficile and C. difficile infection Clin Infect Dis 2008;46 Suppl 1:S4-11.

9. Lima AA, Lyerly DM, Wilkins TD, Innes DJ, Guerrant RL. Effects of Clostridium difficile toxins A and B in rabbit small and large intestine in vivo and on cultured cells in vitro. Infect Immun 1988;56(3):582-8.

10. Lyerly DM, Saum KE, MacDonald DK, Wilkins TD. Effect of Clostridium difficile toxins given intragastrically to animals. Infect Immun 1985;47(2):349-52.

11. Poxton IR, McCoubrey JM, Blair G. The pathogenicity of Clostridium difficile. Clin Microbiol Infect. 2001;7(8):421-7.

12. Vaishnavi C, Kochhar R, Bhasin DK, Thapa BR, Singh K. Detection of Clostridium difficile toxin by an indigenously developed latex agglutination assay. Trop Gastroenterol. 1999;20:33(1)-5.

13. Heard SR, O'Farrell S, Holland D, Crook S, Barnett MJ, Tabaqchali S. The epidemiology of Clostridium difficile with use of typing scheme: nosocomial acquisition and cross-infection among immunocompromised patients $J$ Infect Dis .1986;153:159-62.

14. Kaatz GW, Gitlin SD, Schaberg DR, Wilson KH, Kauffman CA, Seo SM et al. Acquisition of Clostridium difficile from the hospital environment. Am J Epidemiol 1988;127:1289-94.

15. Pillai A, Nelson RL. Probiotics for treatment of Clostridium difficile-associated colitis in adults. Cochrane Database Syst Rev 2008;23(1):CD004611.

16. Dhawan B, Chaudhry R. An update on Clostridium difficile infection. Trop. Gastroenterol 1997;18(4):149-52.

17. Poutanen SM, Simor AE. Clostridium difficile-associated diarrhea in adults. Can Med Assoc J 2004;171(1):51-8.

18. Nelson RL,Kelsey P, Leeman H, Meardon N, Patel H, Paul K, et al. . Antibiotic treatment for Clostridium difficile-associated diarrhea in adults. Cochrane Database Syst Rev 2011;(9):CD004610. 
19. Wilson KH. The microecology of Clostridium difficile. Clin Infect Dis. 1993; 16: S214-8.

20. Akerlund T, Svenungsson B, Lagergren A, Burman LG. Correlation of disease severity with fecal toxin levels in patients with Clostridium difficile-associated diarrhea and distribution of PCR ribotypes and toxin yields in vitro of corresponding isolates. J Clin Microbiol. 2006;44:353-8.

21. McDonald LC, Killgore G, Thompson A, Owens RC Jr, Kazakova SV, Sambol SP et al. An epidemic, toxin gene-variant strain of Clostridium difficile. N Engl J Med. 2005;353(23):243341.

22. Henrich TJ, Krakower D, Bitton A, Yokoe DS. Clinical risk factors for severe Clostridium difficile-associated disease. Emerg Infect Dis. 2009;15(8):415-22.

23. Das R, Feuerstadt P, Brandt LJ. Glucocorticoids are associated with increased risk of short term mortality in hospitalized patients with Clostridium difficile associated disease. Am J Gastroenterol. 2010; 105(9): 2040-9.
24. Bajaj JS, Ananthakrishnan AN, Hafezullah M, Zadvornova Y, Dye A, McGlinley EL et al. Clostridium difficile is associated with poor outcomes in patients with cirrhosis: A national and tertiary center perspective. Am J Gastroenterol. 2010;105(1):106-13.

25. Halvorson SA, Cedfeldt AS, Hunter AJ. Fulminant non-antibiotic associated Clostridium difficile colitis following Salmonella gastroenteritis. J. Gen. Intern Med. 2011; 26(1): 95-7.

26. Dubberke ER, Reske KA, Olsen MA, McDonald LC, Fraser VJ. Short and long term attributable cost of Clostridium difficileassociated disease in non-surgical patients. Clin Infect Dis. 2008;46(4): 497-504.

27. Fekety R. Guidelines for the diagnosis and management of Clostridium difficile-associated diarrhea and colitis. Am J Gastroenterol. 1997;92(5):739-50.

28. Pinto LJF, Alcides APP, Ferreira EO, Avelar KES, Sabra A, Domingues RMCP et al. Incidence and importance of Clostridium difficile in paediatric diarrhea in Brazil. J Med Microbiol 2003;52:1095-9. 


\section{PDF created with pdfFactory Pro trial version www.pdffactory.com}

